\title{
Coronary Artery Thromboses, Stent Thrombosis and Antiphospholipid Antibody Syndrome: Case Report
}

\author{
Augusto Ferreira Correia ${ }^{\mathrm{a}}$, Dinaldo Cavalcanti Oliveira ${ }^{\mathrm{a}}$, b, c, Marcio Sanctos ${ }^{\mathrm{a}}$
}

\begin{abstract}
The antiphospholipid antibody syndrome (APS) is defined by a state of hypercoagulability secondary to an autoimmune disorder. There are evidences that approximately $2.8-5.5 \%$ of cases of acute myocardial infarction (AMI) in young individuals are secondary to APS. In this case report, three coronary artery thromboses occurring within a short period are described. Initially, there was an ST-segment elevation (STEMI) in the presence of coronary artery disease (CAD), with the vessel being treated with stent implantation. Thereafter, a subacute stent thrombosis occurred (high thrombotic load in almost all coronary arteries), which was treated with implantation of two stents. Subsequently, there was a new infarction owing to a new thrombosis in the native coronary artery. The treatment of APS in patients who developed thrombotic events is full anticoagulation from the initial stages maintained throughout life. The standard anticoagulant therapy is administration of vitamin $\mathrm{K}$ antagonists, such as warfarin.
\end{abstract}

Keywords: Acute myocardial infarction; Antiphospholipid antibody syndrome; Coronary artery disease; Thromboses

\section{Introduction}

The antiphospholipid antibody syndrome (APS) is defined by a state of hypercoagulability secondary to an autoimmune disorder. It is associated with thrombotic events in the venous and arterial territories as well as obstetric complications characterized by recurrent fetal losses and increased perinatal morbidity [1].

This syndrome is classified as primary when there is no association with other pathologies or secondary when it is linked to an underlying disease. Its presentation is quite variable, constituting a spectrum of findings ranging from the presence of cutaneous alterations to the involvement of multiple organs,

Manuscript submitted December 17, 2017, accepted January 4, 2018

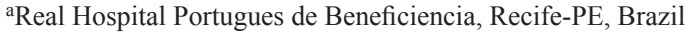

${ }^{b}$ Hospital das Clinicas, Federal University of Pernambuco, Recife-PE, Brazil ${ }^{\mathrm{c} C}$ Corresponding Author: Dinaldo Cavalcanti Oliveira, Real Hospital Portugues de Beneficiencia, Hospital das Clinicas, Federal University of Pernambuco, Recife-PE, Brazil. Email: dinaldo@cardiol.br/dinaldo@pq.cnpq.br

doi: https://doi.org/10.14740/cr661w leading to systemic collapse. Laboratory evaluation reveals the presence of circulating antiphospholipid antibodies (aPLs), which are implicated in the development of endothelial dysfunction among other factors $[1,2]$.

In this context, the development of thrombosis in the coronary arteries is highlighted, and it is estimated that approximately $2.8-5.5 \%$ of cases of acute myocardial infarction (AMI) in young individuals are secondary to APS $[3,4]$.

In APS, the mechanism of myocardial ischemia differs from that which occurs classically in coronary artery disease (CAD), a fact that implies the need for a specific therapeutic approach for patients with this syndrome [4].

Herein, we describe the case of a patient with APS who had AMI with ST-segment elevation (STEMI) on electrocardiogram and subsequent coronary stent thrombosis and new coronary artery occlusion.

\section{Case Report}

N.B., a 65 -year-old married, retired, catholic, and brown man born in a state capital, holding a high school degree, had only systemic arterial hypertension managed with losartan.

He was admitted to the cardiology emergency department with STEMI in the inferior wall. According to the institution's protocol, he was treated with dual antiplatelet aggregation (aspirin and clopidogrel) and adjuvant pharmacological therapy. For $50 \mathrm{~min}$, he was in the hemodynamics laboratory, and subocclusive stenosis was observed in the middle third of the right coronary artery (RCA), which was treated with a percutaneous coronary intervention (stent implant) according to the recommended technique. His condition progressed well, and he was discharged on day 4.

On the fifth day after discharge, the patient was readmitted with a new inferior STEMI and taken to the hemodynamics room, with subacute stent thrombosis being observed in the presence of double antiplatelet aggregation. During the procedure, a large amount of intra-stent thrombi in the RCA as well as in the posterior descending and posterior ventricular branches was observed. In this procedure, two stents were placed (proximal and distal to the already existing stents) with minimal overlapping (Fig. 1), and the recommended pharmacological therapy was administered. Antiplatelet aggregation was modified for acetylsalicylic acid (ASA) and ticagrelor. The thrombi were then aspirated.

Four days after the second ischemic event, the patient had a new STEMI in the same coronary territory. During coronary angiography, another thrombotic event was observed in the 

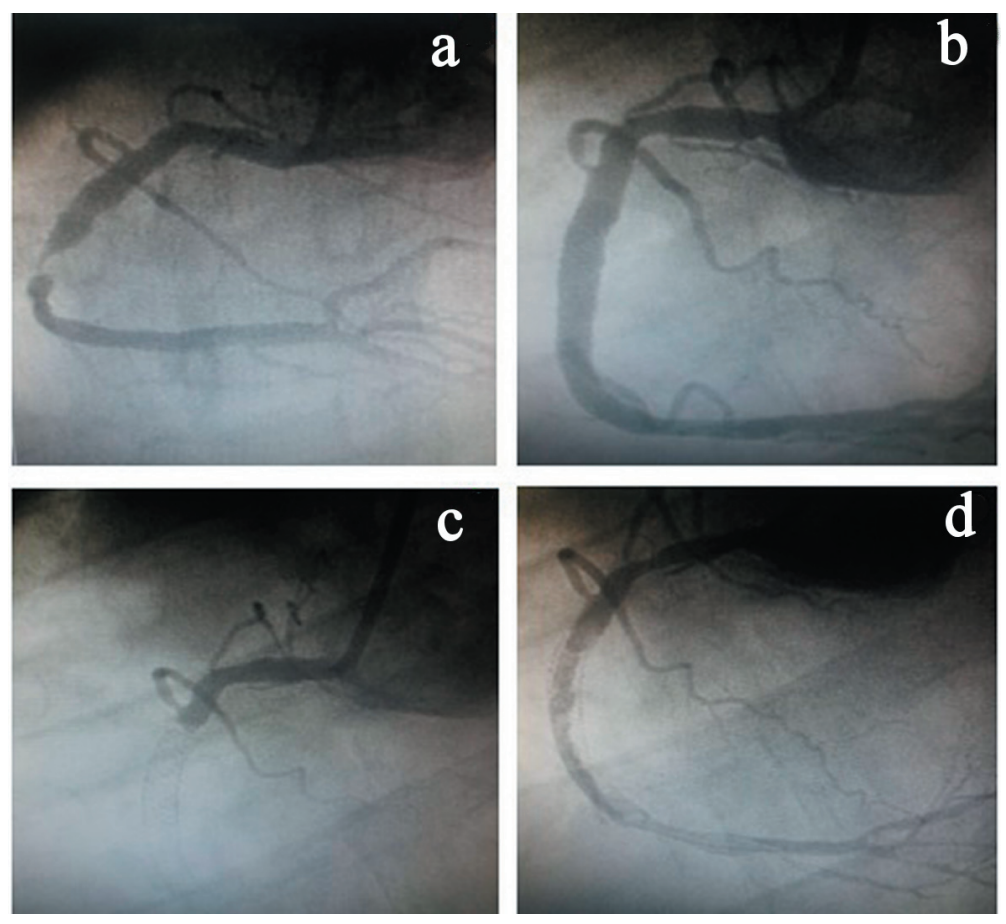

Figure 1. (a) Stenosis in the right coronary artery (RCA). (b) RCA angiography after stent implantation. (c) Stent thrombosis. (d) Large amount of thrombus in the RCA.

RCA where there was no stent. As such, a new percutaneous coronary intervention was performed, but without success. Thus, the vessel remained occluded at the end of the procedure because there was no antegrade flow at any time after the various insufflations using balloon catheters (Fig. 2).

The patient became clinically stable and asymptomatic and was discharged from the hospital on the fifth day and referred to a specialist for thrombophilia research.

During the laboratory evaluation, there was no significant change in the results, except for high titers of an anticardiolipin antibody IgG. This test result was positive at two dosages with an interval greater than 12 weeks. There was no evidence of any other serum markers suggesting other associated pathologies.

As such, primary APS was diagnosed, and oral anticoagulation with early outpatient follow-up was indicated.

\section{Discussion}

In this case report, three coronary artery thromboses occurring within a short period are described. Initially, there was an STEMI in the presence of CAD, with the vessel being treated with stent implantation. Thereafter, a subacute stent thrombosis occurred (high thrombotic load in almost all coronary arteries), which was treated with implantation of two stents. Subsequently, there was a new infarction owing to a new thrombosis in the native coronary artery.

In view of the patient's atypical evolution after the procedures, associated diseases that contributed to the events were investigated. Hence, APS was diagnosed.

In APS, aPLs bind to the membrane receptors of endothe- lial cells, resulting in an increased risk of thrombosis, accelerated atherosclerosis, AMI, and stroke [5, 6].

The inhibition of aPL-mediated endothelial nitric oxide synthase (eNOS) enzyme activity is the molecular basis of endothelial dysfunction, increased leukocyte cell adhesion, and thrombus formation. The binding of circulating aPLs to the $\beta 2$ GPI receptor I domain on endothelial cells induces their dimerization. The subsequent interaction between the dimerized $\beta 2$ GPI and apolipoprotein E receptor 2 induces eNOS inhibition, increased expression of endothelial adhesion molecules, and increased endothelin-1 production and tissue factor, corroborating thrombus formation [7].

The appearance of premature atherosclerosis in patients with APS is associated with the pro-inflammatory state immunomediated by the aPLs, with alteration of subclinical markers of cardiovascular disease, such as increased medial-intimal thickness of the carotid arteries and changes in the ankle-brachial index [1].

The incidence of AMI cases secondary to APS ranges from $2.8-5.5 \%$, and this syndrome should be considered in patients younger than 55 years and with an atypical progression $[3,4]$. In situations in which cardiovascular risk factors coexist, patient stratification should be sought for other etiologies $[1,4]$.

The patient in this report only had hypertension, without other classic risk factors related to CAD, but fulfilled the diagnostic criteria for APS (serologic marker and documented thrombotic event).

AMI in patients with APS usually occurs in the fourth decade of life, being more common in women and without changes in the angiographic study results $[3,4]$. The case described differs from the classical epidemiology because the patient 

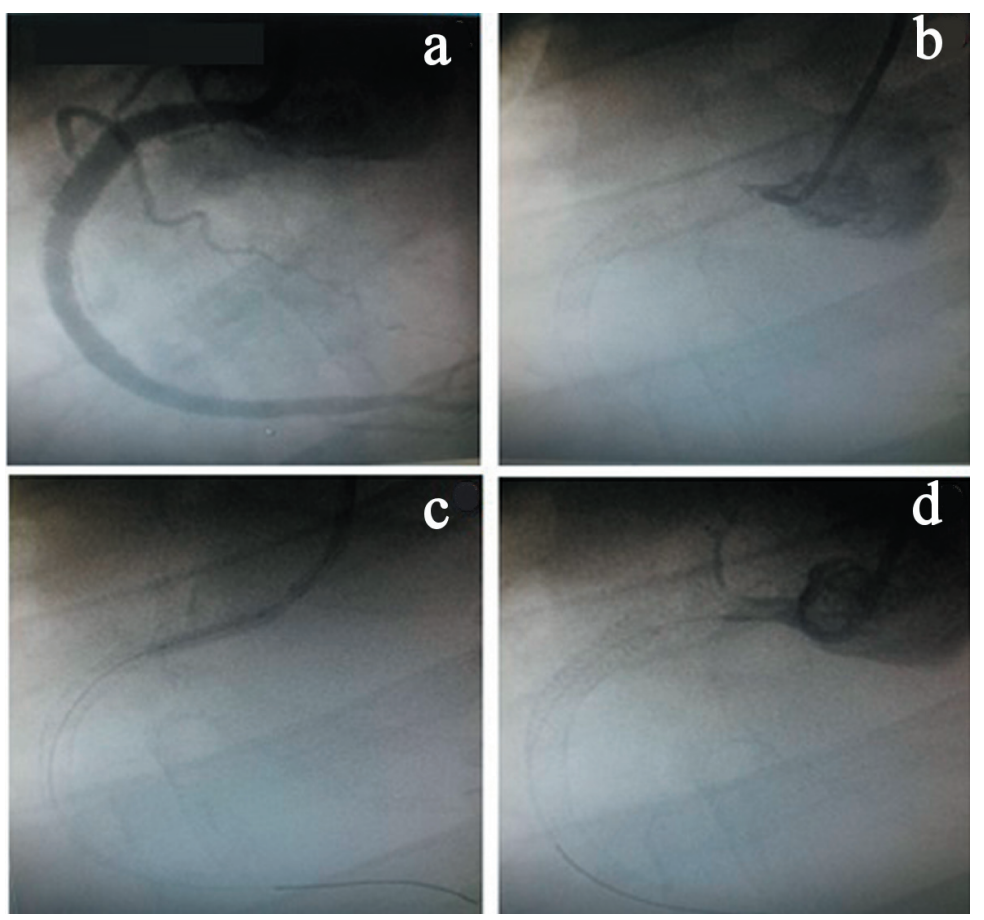

Figure 2. (a) Right coronary artery angiography (RCA) after stent implantation. (b) RCA occlusion (origin). (c) Inflated balloon catheter in the RCA origin. (d) RCA occluded.

was a man in the seventh decade of life and had no clinical, serological, or family history of autoimmunity.

The treatment of AMI due to CAD is based on coronary reperfusion (more effective with stent implantation) and on adjunct pharmacological therapy; one of its pillars is double antiplatelet aggregation. After percutaneous revascularization, full anticoagulation is not recommended [4].

Conversely, the treatment of APS in patients who developed thrombotic events is full anticoagulation from the initial stages maintained throughout life [8]. The standard anticoagulant therapy is administration of vitamin $\mathrm{K}$ antagonists, such as warfarin. No new anticoagulant was superior to warfarin in preventing new events [9].

Reduction in the serum levels of aPLs through immunomodulation, with the use of corticosteroids, immunosuppressant or plasmapheresis, may be necessary in cases of secondary APS $[2,8,9]$.

In the patient described here, the diagnosis of this syndrome was not known; therefore, it was treated in accordance with the guidelines. However, we believe that the presence of metal rods within the coronary artery in contact with the endothelium and blood in the absence of full anticoagulation was a potent thrombogenic factor; this contributes to the imbalance between the organism's coagulation and fibrinolysis systems. Further, we also believe that there was a thrombogenic joint effect of atherosclerosis and APS.

\section{Conclusions}

In this case, the most effective therapy was possibly not offered, since he had APS with a pathophysiology presenting peculiar aspects related to the induction of ischemia and/or necrosis.

It is necessary that the multidisciplinary teams taking care of patients with AMI have a high degree of suspicion of the causes other than classic CAD, especially when these patients do not present a customary clinical evolution. In this context, APS should be considered.

\section{References}

1. Corban MT, Duarte-Garcia A, McBane RD, Matteson EL, Lerman LO, Lerman A. Antiphospholipid syndrome: role of vascular endothelial cells and implications for risk stratification and targeted therapeutics. J Am Coll Cardiol. 2017;69(18):2317-2330.

2. Cervera R. Antiphospholipid syndrome. Thromb Res. 2017;151(Suppl 1):S43-S47.

3. Cervera R, Serrano R, Pons-Estel GJ, Ceberio-Hualde L, Shoenfeld Y, de Ramon E, Buonaiuto V, et al. Morbidity and mortality in the antiphospholipid syndrome during a 10-year period: a multicentre prospective study of 1000 patients. Ann Rheum Dis. 2015;74(6):1011-1018.

4. Nazir S, Tachamo N, Lohani S, Hingorani R, Poudel DR, Donato A. Acute myocardial infarction and antiphospholipid antibody syndrome: a systematic review. Coron Artery Dis. 2017;28(4):332-335.

5. Petri M. Epidemiology of the antiphospholipid antibody syndrome. J Autoimmun. 2000;15(2):145-151.

6. Mineo C. Inhibition of nitric oxide and antiphospholipid antibody-mediated thrombosis. Curr Rheumatol Rep. 
2013;15(5):324.

7. Ninivaggi M, Kelchtermans H, Lindhout T, de Laat B. Conformation of beta2glycoprotein I and its effect on coagulation. Thromb Res. 2012;130(Suppl 1):S33-36.

8. Arachchillage DRJ, Laffan M. Pathogenesis and management of antiphospholipid syndrome. Br J Haematol. 2017;178(2):181-195.
9. Ruiz-Irastorza G, Cuadrado MJ, Ruiz-Arruza I, Brey R, Crowther M, Derksen R, Erkan D, et al. Evidence-based recommendations for the prevention and long-term management of thrombosis in antiphospholipid antibodypositive patients: report of a task force at the 13th International Congress on antiphospholipid antibodies. Lupus. 2011;20(2):206-218. 